Research report

\title{
Dynamics of host and graft after cell sheet transplantation: Basic study for the application of amyotrophic lateral sclerosis
}

\author{
Mami Nakanishi ${ }^{\mathrm{a}}$, Yasuhiro Watanabe ${ }^{\mathrm{a}, *}$, Naoto Honda ${ }^{\mathrm{a}}$, Mio Une ${ }^{\mathrm{a}}$, Kanako Kazuki ${ }^{\mathrm{b}}$, \\ Yasuhiro Kazuki $^{\mathrm{b}}$, Tomoya Terashima ${ }^{\mathrm{c}}$, Miwako Katagi ${ }^{\mathrm{c}}$, Kenji Nakashima ${ }^{\mathrm{d}}$, Ritsuko Hanajima ${ }^{\mathrm{a}}$ \\ ${ }^{a}$ Division of Neurology, Department of Brain and Neurosciences, Faculty of Medicine, Tottori University, Japan \\ ${ }^{\mathrm{b}}$ Division of Humanized Model Animal Development, Chromosome Engineering Research Center, Tottori University, Japan \\ ${ }^{\mathrm{c}}$ Department of Stem Cell Biology and Regenerative Medicine, Shiga University of Medical Science, Japan \\ ${ }^{\mathrm{d}}$ Department of Neurology, National Hospital Organization, Matsue Medical Center, Japan
}

\section{H I G H L I G H T S}

- To improve the survival of transplanted cells in host tissues is a key for successful transplantation.

- Transplanting donor cells as a sheet structure, we successfully prolonged the survival of donor cells.

- There was an encouraging trend in the delayed onset of symptoms and increased lifespan in ALS model mice.

\section{A R T I C L E I N F O}

\section{Keywords:}

Amyotrophic lateral sclerosis

Cell transplantation

Human artificial chromosome

Mesenchymal stem cell

Luciferases

Tissue engineering

\begin{abstract}
A B S T R A C T
Stem cells offer great hope for the therapy of neurological disorders. Using a human artificial chromosome (HAC), we generated modified mesenchymal stem cells (MSCs), termed HAC-MSC that express 3 growth factors and 2 marker proteins including luciferase, and previously demonstrated that intrathecal administration of HACMSCs extended the lifespan in a mouse model of amyotrophic lateral sclerosis (ALS). However, donor cells disappeared rapidly after transplantation. To overcome this poor survival, we transplanted the HAC-MSCs as a sheet structure which retained the extracellular matrix. We investigated, here, whether cell sheet showed a longer survival than intrathecal administration. Also, the therapeutic effects on ALS model mice were examined.

In vivo imaging showed that luciferase signals increased immediately after transplantation up to 7 days, and these signals were sustained for up to 14 days. In contrast, following intrathecal administration, signals were drastically decreased by day 3 . Moreover, cell sheet transplantation successfully prolonged the survival of donor HAC-MSCs. Cell sheet transplantation increased the level of p-Akt at the graft area. Pathologically, none of the donor cells differentiated into neurons, astrocytes or microglial cells. When the cell sheet was transplanted into ALS model mice, there was an encouraging trend in the delayed onset of symptoms and increased lifespan. If each group was subdivided into rapid and slow progressors based on cut-off values for respective median survival, the survival of rapid progressors differed significantly between groups (treated vs. sham-operated $=145.4 \pm 1.4$ vs. $139.2 \pm 1.2$ ). The effect of HAC-MSC sheet transplantation still has a temporally narrow therapeutic window.

Further improvement could be achieved by optimization of the transplantation conditions, e.g. co-transplantation of HAC-MSCs with endothelial progenitor cells.
\end{abstract}

\section{Introduction}

Cell-based therapies have garnered much attention as novel approaches to the cure of neurodegenerative diseases such as Parkinson's disease, amyotrophic lateral sclerosis (ALS), and neural injury (Coatti et al., 2015; Hsuan et al., 2016; Joyce et al., 2010). The primary aim of these strategies is to delay or prevent neuronal cell death. Recent studies have suggested that stem cell transplantation exerts therapeutic benefits via several mechanisms including not only the replenishment of lost neurons but also through cytoprotection from secreted

\footnotetext{
* Corresponding author at: Division of Neurology, Department of Brain and Neurosciences, Faculty of Medicine, Tottori University, 36-1 Nishi-cho, Yonago 6838504, Japan.

E-mail address: yawatana@tottori-u.ac.jp (Y. Watanabe).
} 


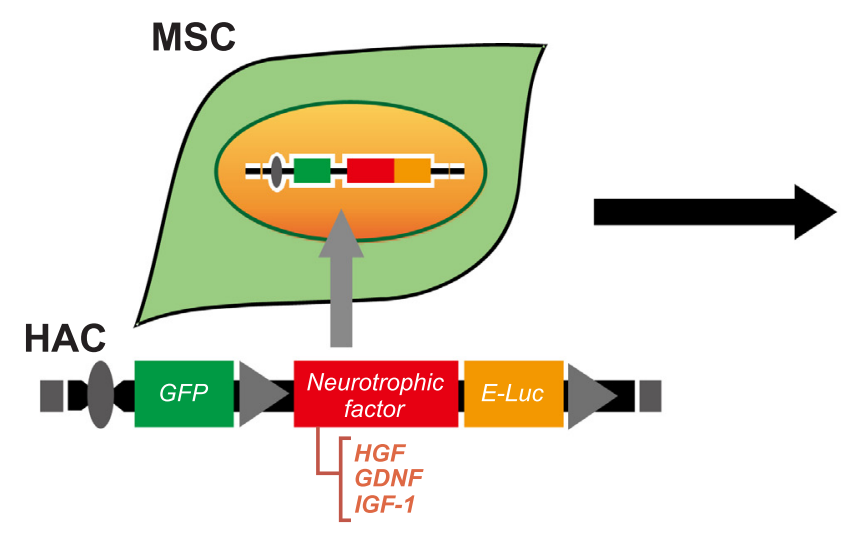

Cell culture

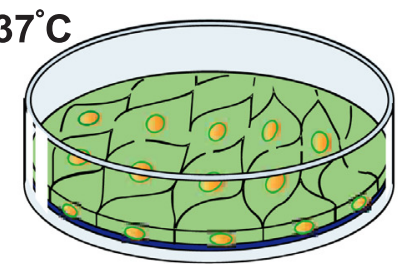

Temperature-sensitive dish

$25^{\circ} \mathrm{C}$

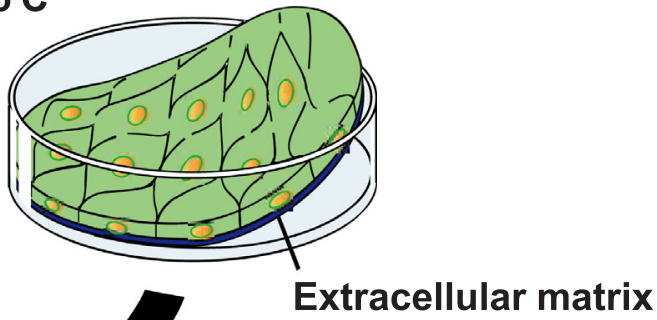

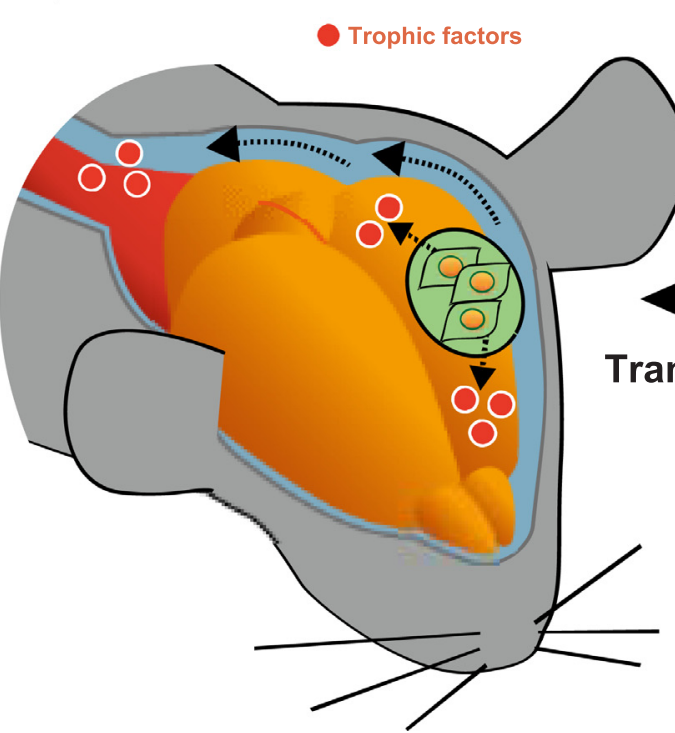

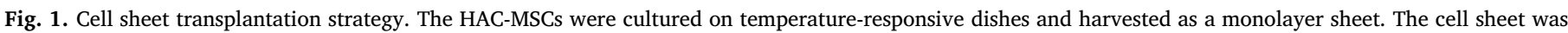

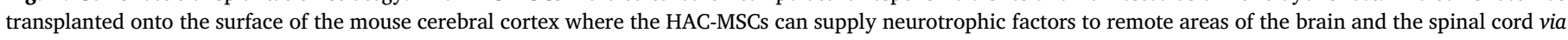
the CSF. If applied to human ALS patients, the cell sheet can be transplanted onto surface of the ventral side of the spinal cord.

neurotrophic factors (Gnecchi et al., 2016; Lunn et al., 2009). While such therapeutic approaches have shown positive effects in basic research using animal models, much work and refinement is needed before they are ready for use in humans (Gothelf et al., 2014; Mazzini et al., 2010; Oh et al., 2015; Sykova et al., 2017). Another promising strategy is the administration of neurotrophic factors. However, there are several hurdles to this approach, such as poor penetration of the trophic factors into the central nervous system (CNS) by the presence of the blood brain barrier (BBB), systemic side effects, short half-life within the blood stream, and the high cost of large scale production of such factors. Thus, neurotrophic factors have yet to be approved for clinical use (Henriques et al., 2010; Sampaio et al., 2017).

To overcome these challenges, we have established a mesenchymal stem cell (MSC) line that expresses high levels of neurotrophic factors using a human artificial chromosome (HAC) system, termed HAC-MSCs (Watanabe et al., 2015). MSCs, originated from mesoderm, are reported to promote tissue rescue/repair via paracrine activity, exosomes, immunomodulation activity, and so on (Mazzini et al., 2010). These HACMSCs simultaneously express three potent neurotrophic factors for the survival of motor neurons: hepatocyte growth factor (HGF), glial cell line-derived neurotrophic factor (GDNF) and insulin-like growth factor1 (IGF-1) as well as reporter proteins: green fluorescent protein (GFP), Emerald luciferase (E-Luc; Toyobo, Osaka, Japan) (Watanabe et al., 2015). In addition to the trophic effect of MSCs themselves, HAC-MSCs can act as a vector for neurotrophic factors to the CNS. Previously, we showed that transplantation of HAC-MSCs via the fourth cerebral ventricle into ALS model mice resulted in a significant improvement in lifespan (Watanabe et al., 2015). However, we also revealed that the number of transplanted cells dramatically decreased with time, and almost none of the cells survived more than one week after transplantation. Thus, the ability to prolong the survival of donor cells after transplantation is a key factor for improving their therapeutic efficacy. This massive donor cell death might occur due to the sudden environmental change from culture conditions to the surface of the host brain tissue which is surrounded by cerebrospinal fluid (CSF); these changes include a lack of blood supply, oxidative stress, and the sugar and protein-poor environment of the CSF, among others (Sart et al., 2014).

Cell sheet engineering is a promising approach in stem cell transplantation. Conventional cell harvesting methods using a proteolytic enzyme, such as trypsin, cause varying degrees of damage and even pose the risk of phenotypic change. In contrast, a cell sheet is harvested without the use of proteolytic enzymes, thus maintaining the network of extracellular matrix. This could facilitate adhesion to host tissues and help minimize the loss of donor cells after transplantation. In addition, retention of the cell-cell structural network among donor cells reduces their free migration, which, in certain situations, enhances the safety of transplantation. There are several protocols for preparing cell sheets; these include coating the culture dish with fibrin or temperature- 
sensitive polymer, poly ( $\mathrm{N}$-isopropylacrylamide), and vitamin $\mathrm{C}$ treatment. Temperature-sensitive culture dishes are widely used as the cell sheet can easily be collected by altering the temperature from $37^{\circ} \mathrm{C}$ to $30^{\circ} \mathrm{C}$ or below. MSC sheets have been widely used in the field of regenerative medicine for cardiac muscle and bone regeneration (Chen et al., 2015; Miyahara et al., 2006; Ouyang et al., 2006). In the field of CNS disorders, Ito et al. recently reported the application of a bone marrow-derived MSC sheet to a rat stroke model (Ito et al., 2017). However, there have yet to be any reports of the application of cell sheets for neurodegenerative diseases. The cerebral cortex can be more easily exposed than the spinal cord and its surface is larger and thus more suitable for transplanting a cell sheet. Moreover, it is thought that neurotrophic factors secreted from the transplanted HAC-MSCs get distributed throughout the CNS via the CSF. Thus, the cell sheet likely acts as a continuous source of neurotrophic factors to the CNS along with the neurotrophic effects of the MSCs themselves. This therapeutic strategy would, therefore, be suitable and valid for the treatment of neurodegenerative diseases especially ALS, as ALS-associated lesions are broadly distributed from the motor cortex to the anterior horn of the lumbar spinal cord.

In this study, HAC-MSC cell sheets were prepared and transplanted onto the surface of the mouse cerebral cortex. The survival time course of the engrafted cells was monitored and compared to survival after intrathecal administration using in vivo live imaging. Any phenotypic alterations of the donor cells and host tissues after transplantation were observed histologically. We also examined the clinical efficacy of HACMSC sheet transplantation in a mouse model of ALS.

\section{Results}

\subsection{Cell sheet transplantation in mice}

The HAC-MSCs were harvested as a cell sheet which contained about $3 \times 10^{5}$ cells/sheet. The left cerebral cortex of each mouse was surgically exposed and the cell sheet was placed onto this region (Fig. 1). After transplantation, neither infection at the surgical site, paralyses nor abnormal behaviors were observed. One day after transplantation a strong green fluorescent signal derived from the HAC-MSCs was detected (Figure Supplementary 1). One week after transplantation, the HAC-MSCs remained at the transplant site and the layered structure was maintained (Figure Supplementary 1). Donor cell migration into the deep structures of the recipient was not observed.

\subsection{Longitudinal observations of HAC-MSCs using bioluminescence imaging (BLI)}

We first monitored in vitro luminescence values using cultured HACMSCs (Fig. 2A), and a linear correlation between the predetermined number of cells and the luminescence values was confirmed (Fig. 2B).

Next, in vivo BLI analyses showed detectable signals from the donor cells until day 14 after sheet transplantation in the mouse left cerebral cortex (Fig. 2C). The signals increased immediately after transplantation (day 0 ) up to day 7 , and were particularly strong on day 7 (576\% \pm 266 compared to day 0; Fig. 2D).

The BLI signals were also monitored after intrathecal injection of a suspension of HAC-MSCs into the fourth ventricle (Fig. 2E). Using the same sensitivity settings as for the cell sheet transplantation, the BLI signal value after intrathecal injection was undetectable even immediately after transplantation. By extending the exposure time and increasing the detectable resolution, BLI signals became detectable (Fig. 2E); however, they decreased rapidly between day 3 and 7 (Fig. 2F). Only two mice out of 4 showed weak signals on day 14 .

Interestingly, in the sheet transplantation, the BLI signals increased by almost 6 -fold after transplantation from day 0 to 7 . However, by histological observation, we were unable to confirm a marked increase in the number of transplanted cells through the first week (Figure
Supplementary 1). This discrepancy between the increased BLI signal and the lack of histological confirmation might be explained by the poor access of the donor cells to luciferin. Specifically, in the BLI analysis immediately after transplantation, premature engrafted donor cells cannot access the luciferin via the blood stream or the cerebrospinal fluid.

\subsection{Upregulation of p-Akt by HAC-MSC sheet transplantation}

Protein levels of Akt, a major transcription factor in cell survival pathways, were determined to confirm the neuroprotective effects of HAC-MSCs in vivo. Total Akt (t-Akt) and active phosphorylated Akt (pAkt) were measured, and each value was normalized with $\alpha$-Tubulin. The transplanted brain region, including the donor cells and the host tissue, was excised, and immuno-blot analyses were performed using anti-t-Akt, anti-p-Akt and anti- $\alpha$-tubulin antibodies. Non-treated animal brain tissues were used as controls. Results showed a significant increase in p-Akt after HAC-MSC sheet transplantation (Fig. 3). The expression of t-Akt and the p-Akt/t-Akt ratio also showed an increasing trend, but did not reach statistical significance.

\subsection{Therapeutic impact in a mouse model of ALS}

To examine the efficacy of the HAC-MSCs, we transplanted cell sheets into a mouse model of ALS, SOD $1{ }^{\mathrm{G} 93 \mathrm{~A}}$ mice, at 100 days of age during the presymptomatic phase ( $n=24 ; 12$ males, 12 females). For controls, sham-operations were performed ( $n=24 ; 12$ males, 12 females). There was a beneficial tendency for delayed onset of disease (Fig. $4 \mathrm{~A}$; treated vs. control $=137.1 \pm 1.5$ vs. $134.8 \pm 1.6$, $p=0.73$ ), age at death (Fig. 4B; treated vs. control $=149.9 \pm 1.3$ vs. $146.8 \pm 1.9, p=0.74$ ), and improvement in hindlimb reflex at 17 weeks (Fig. 4C; treated vs. control $=1.73 \pm 0.07$ vs. $1.55 \pm 0.07$, $p=0.089$ ); however, these improvements were not statistically significant. There were no differences in body weight or disease duration between the groups (Fig. 4D and E).

When we compared median survival in both groups, the transplanted group survived 150 days and control survival was 144 days. Considering that we transplanted the cell sheets into ALS model mice at 100 days and the transplanted cells survive up to 14 days, we subdivided each group into rapid and slow progressors based on cut-off values for respective median survival, the survival of rapid progressors differed significantly between groups (Fig. 4F; treated vs. control $=145.4 \pm 1.4$ vs. $139.2 \pm 1.2, p<0.001$ ). Although the HACMSC sheets remain in the host tissue for at least two weeks, these results suggest that the effect of HAC-MSC sheet transplantation still has a temporally narrow therapeutic window.

\subsection{Phenotypic changes in donor and host after cell sheet transplantation}

Brains were collected 7 days after transplantation to obtain coronal sections of the cerebral cortex. The localization of GFP fluorescence derived from the HAC-MSCs and the expression of neural cell markers was examined using immunohistochemical staining. Multilayered green donor cells were observed on the brain surface (Fig. 5A-H). These layers were negative for DCX, NeuN and GFAP (Fig. 5A, C and E); however, they were seemingly positive for Iba1 (Fig. 5G). High-magnification confocal microscopy (Fig. 5I) showed the presence of Iba1positive cells (red) around the HAC-MSCs (green); these signals did not overlap with that of the HAC-MSCs, suggesting that the Iba1-positive cells originated from the host. We next examined phenotypic markers for microglias; whether they were activated into neurotoxic M1: nitric oxide synthease 2 (NOS2) positive or neuroprotective M2: arginase 1 (Arg1) positive. As a result, these infiltrated microglias are partly M2 phenotype cells but some population was M1 marker positive (Fig. 6). These M1 activated microglias might play a role in the rejection of the HAC-MSC sheet. M1 and M2 marker expressing cells locate in the HAC- 
A

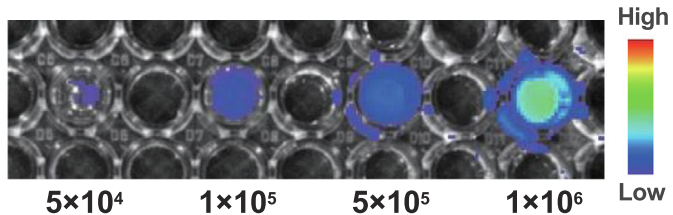

C
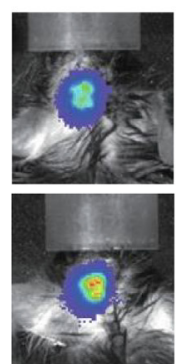

Day0

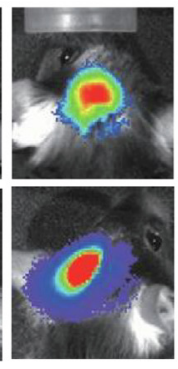

Day3

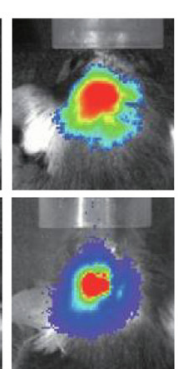

Day7

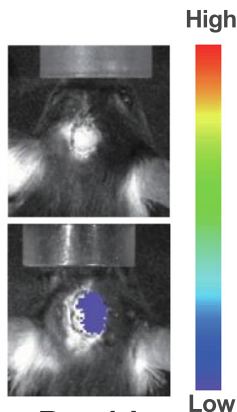

Day14

High

B

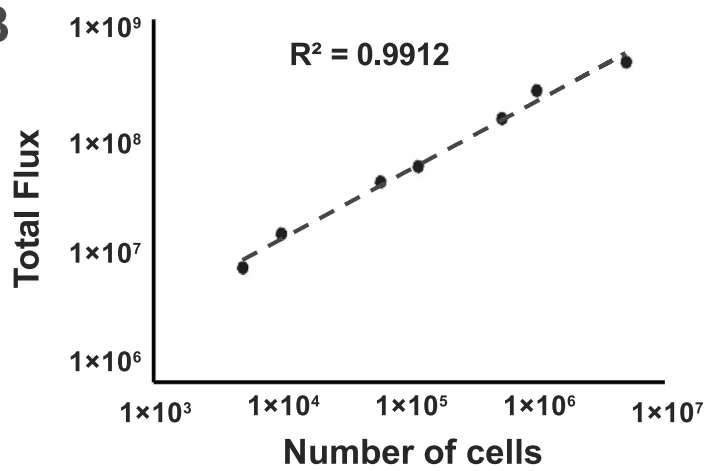

E
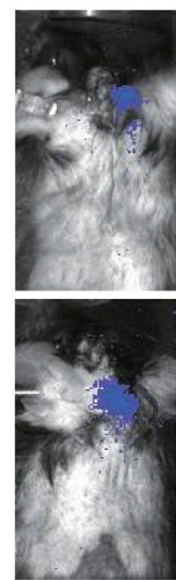

Day0

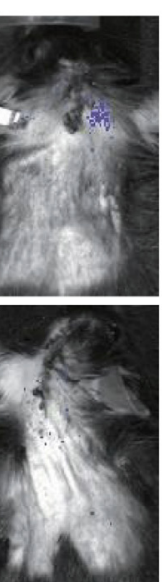

Day3

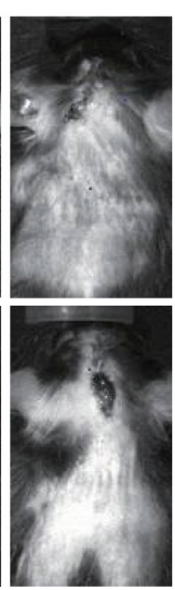

Day7

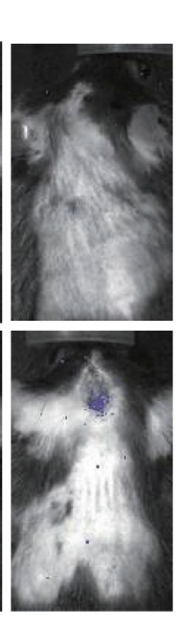

Day14
High

$\mathbf{F}$
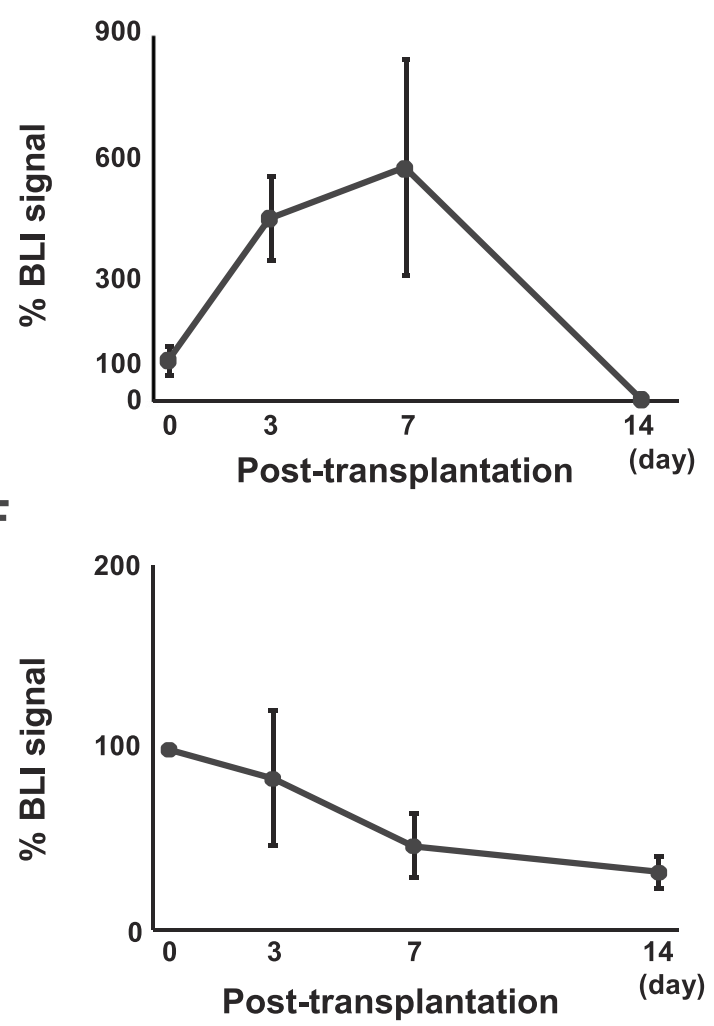

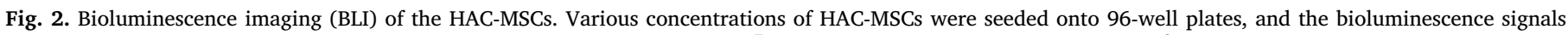

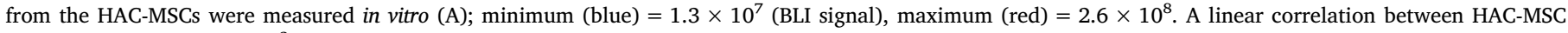

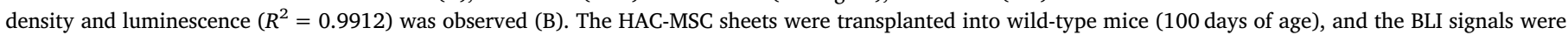

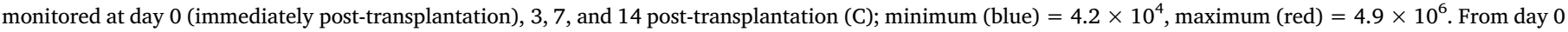

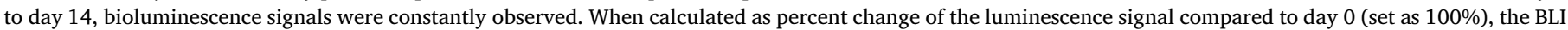

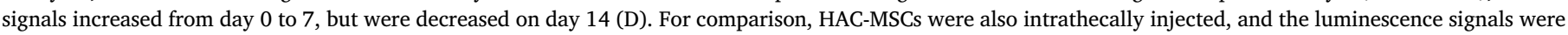

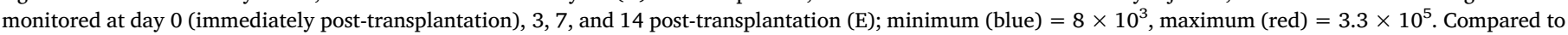
day 0 , the signals decreased throughout the observation period (F).

\section{MSC transplanted region.}

We next observed changes in the host brain tissue after HAC-MSC sheet transplantation. Within the host brain tissues adjacent to the transplanted area, GFAP and Iba1 immunoreactivity was dramatically increased in the transplanted group as well as in the sham-operated group, indicating that host astrocytes and microglial cells were activated by the surgical procedure (Figure Supplementary 2).

\section{Discussion}

\subsection{Differentiation of HAC-MSCs}

MSCs are multipotent cells capable of differentiating into adipocytes, chondrocytes and osteoblasts. In addition, many studies have reported their differentiation into non-mesodermal cells such as neurons and glia (Blondheim et al., 2006; Foudah et al., 2012, 2013). The therapeutic potential of MSCs has been well established in experimental models of stroke (Borlongan, 2019), attributed in part to the secretion of neurotrophic factors (Toyoshima et al., 2015) and their capacity to 

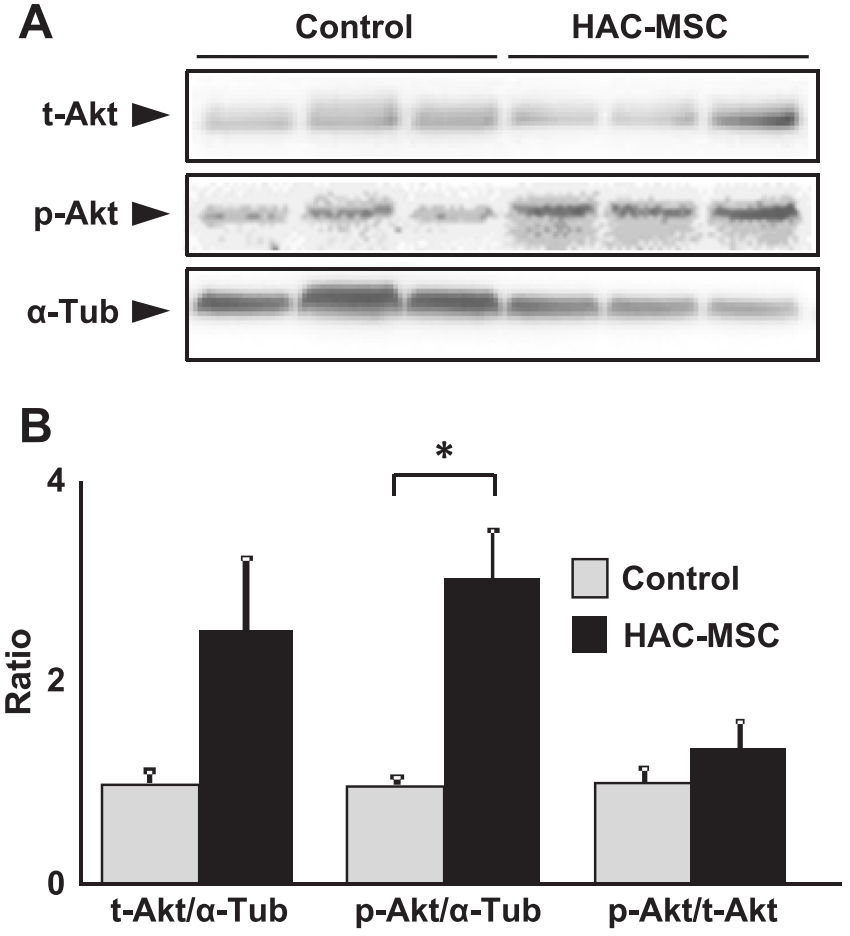

Fig. 3. HAC-MSC transplantation upregulates p-Akt. Seven days after transplantation, the transplanted region was excised, and the amount of total AKT (tAkt), phosphorylated AKT (p-Akt) and $\alpha$-tubulin ( $\alpha$-Tub) were determined by western blot (A). The protein levels of t-Akt and p-Akt (measured values were normalized to those of $\alpha$-tubulin) and the p-Akt/t-Akt ratio were quantified (B). There was a significant increase in p-Akt in the HAC-MSC transplanted group ( $n=4$, black) compared to the non-treated group ( $n=5$, grey; $*: p<0.05$ ). The amount of t-Akt and the p-Akt/t-Akt ratio showed an increasing trend but did not reach significance.

attenuate chronic inflammation (Acosta et al., 2015). Further the neuroprotective effects of MSCs are mediated in part by anti-apoptotic mechanisms (Zhang et al., 2019).

Consistent with our previous report (Watanabe et al., 2015), the transplanted HAC-MSCs did not show any microglial, astrocytic or neuronal phenotypes. Doublecortin (DCX), a marker for neuronal precursor cells and immature neurons, was also negative in the present study. However, in other transplantation experiments using mouse models of neurodegenerative disease, transplanted MSCs have been reported to exhibit astrocytic and neuron-like phenotypes after transplantation (Chen et al., 2017; Vercelli et al., 2008), suggesting the possibility of neuronal differentiation of MSCs in vivo. Transplantation of MSCs into a mouse model of Huntington's disease, however, did not result in donor cell differentiation into neurons, astrocytes, nor microglia (Lescaudron et al., 2003). Moreover, Ito et al. reported that after cell sheet transplantation into a rat model of cerebral infarct, donor MSCs were negative for NeuN and GFAP (Ito et al., 2017), although they did not discuss the presence of microglial phenotypes. These studies suggest that the phenotype of MSCs after transplantation might be affected by the microenvironment around the graft site and the state of the cells at the time of transplantation.

\subsection{Glial activation evoked by HAC-MSC sheet transplantation}

The cell sheet transplantation surgery, which is accompanied by invasive procedures including resection of the dura and exposure of the cerebral cortex, can cause varying degrees of brain injury, as was observed in mice which received a sham-operation alone in our experiment. De Vocht et al. indicated that transplantation of MSCs into the CNS can cause neuroinflammation at the graft site with recruitment of astrocytes and microglia (De Vocht et al., 2013), consistent with our immunohistochemical observations. Microglial activation has both neurotoxic and neuroprotective effects during neurodegeneration (Endo et al., 2015). However, as our transplantation experiment was performed in wild-type mice without any underlying progression of neurodegenerative processes, microglial M2 activation at the transplantation site would be mainly caused by active processes in the host tissue as a reaction to the surgical invasion and the activation of reparative pathways. Intriguingly, we also observed that numerous microglial cells had infiltrated into the cell sheet structure, and these activated microglias were not only M2 but also M1 population. We did not observe such phenomena in intrathecal transplantation, possibly due to the low number of surviving cells. Cell sheet transplantation offers the unique opportunity to observe the host-graft relationship in the CNS. Even though we conducted the transplantation experiments using the immunosuppressant FK506, we cannot exclude the possibility that activated microglia, especially M1 phenotype might negatively affect the survival of donor cells. If so, it will be necessary to modulate neuroinflammation and upregulate neuroprotection in order to further increase the potential therapeutic effects. The HAC vector has several advantages in this regard; the vector can harbor multiple genes, and is stably maintained in daughter cells during cell proliferation. A further understanding of neuroinflammatory mechanisms could potentially allow us to introduce modulators of inflammation into the donor cells.

\subsection{Therapeutic efficacy}

In this study, we confirmed that cell sheet transplantation extended the survival of grafted cells compared to the injection of a cell suspension. For more than 2 weeks post-transplantation, the grafted cells were visible in vivo by luciferase luminescence imaging. In the transplanted area, the increased expression of p-Akt as well as t-Akt was observed compared to non-treated animals, demonstrating the molecular basis of the cytoprotective effects exerted by the transplantation of cell sheets as well as the trophic factors introduced into the HAC vector. Furthermore, HAC-MSC sheet transplantation into ALS mice in vivo showed a tendency toward extending the survival of the mice.

Recent studies have indicated that in G93A ALS rats, the dysfunction of upper motor neurons occurs during the pre-symptomatic phase, prior to the loss of spinal lower motor neurons (Thomsen et al., 2014). If the preceding upper motor neuron dysfunction contributes to lower motor neuron degeneration, and earlier intervention to upper motor neurons can alter the course of lower motor neuron degeneration, this would validate our therapeutic strategy. Similar to that rat study, in the present study we transplanted HAC-MSC sheets onto the surface of the cerebral cortex during the pre-symptomatic phase in G93A mice.

In this study, we transplanted a cell sheet consisting of $3 \times 10^{5}$ HAC-MSCs/sheet due to the spatial restrictions of the mouse cerebral surface. In the case of intrathecal injection via the fourth cerebral ventricle, the number of transplanted cells was $1 \times 10^{6}$. Thus far, cell sheet transplantation showed promising donor cell survival even transplanted with fewer cells compare to intrathecal transplantation. On the other hand, the lack of significance of HAC-MSC sheet on ALS model mice is undeniable. This is due to, first, we used only small number of cells for cell sheet transplantation, and second, still short survival of the cell sheet. In the first case, we may expand cell number by utilizing a multilayered cell sheet. And second, we may extend the cell sheet survival by heterogeneous cell transplantation, e.g. HACMCSs combined with vascular endothelial progenitor cells, which could promote a more rapid blood supply after transplantation (donor modulation); otherwise by controlling microglial M1 and M2 phenotypes or regulating total microglial populations, we may extend the survival of HAC-MSC sheet (host modulation). Alternatively, cell sheet transplantation and intrathecal injection are not necessarily mutually exclusive. During intrathecal injection, injected cells are dispersed throughout the spinal cord (Morita et al., 2008) and in the case of spinal cord injury, 
A Onset

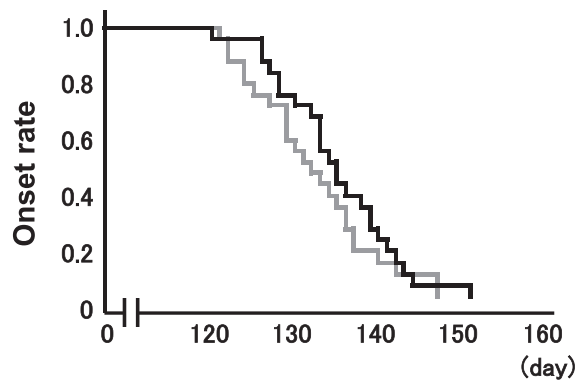

C Hindlimb reflex

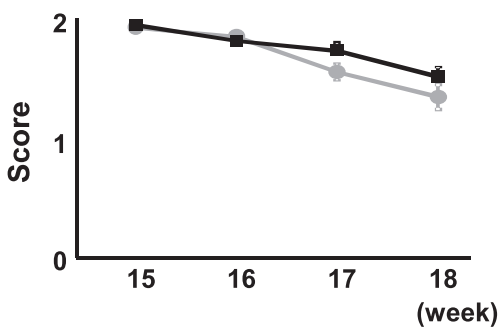

\section{E Disease duration}

(day)

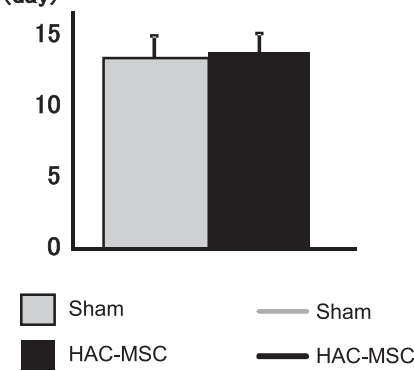

B Endpoint

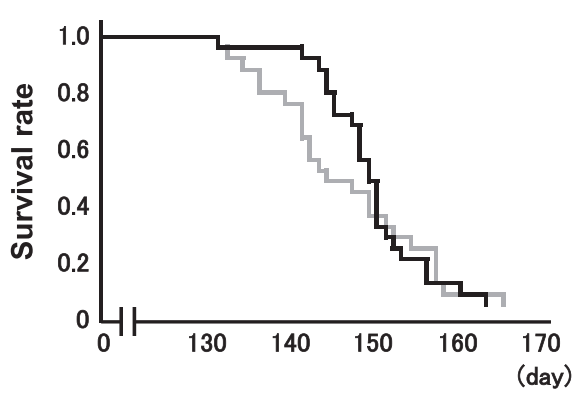

D Body weight

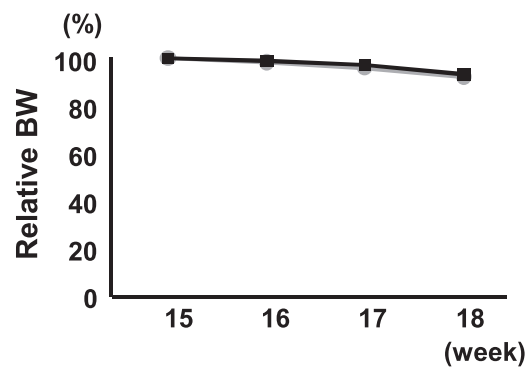

\section{F Rapid progressor endpoint}

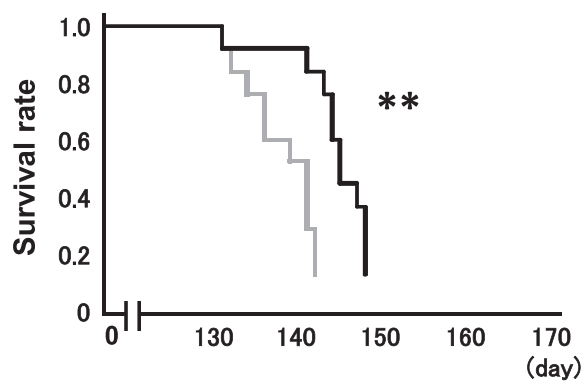

Fig. 4. Evaluation of ALS model mice transplanted with HAC-MSC sheets. HAC-MSC sheet transplantation or a sham operation was performed on ALS model mice. Age of onset (A) and death (B), hindlimb reflex score (C), body weight (D), and disease duration (E) were assessed. In the treated group, a beneficial tendency was observed for the age of death (B; treated vs. control $=149.9 \pm 1.3$ vs. $146.8 \pm 1.9$ ) and the day of onset (A; treated vs. control $=137.1 \pm 1.5$ vs. $134.8 \pm 1.6$ ); however, neither of these improvements reached statistical significance. There were no differences in hindlimb reflex scores (C), weight change (D) nor disease duration (E). When each group was subdivided into rapid and slow progressors based on cut-off values for respective median survival, the survival of rapid progressors differed significantly between groups (F; treated vs. control $=145.4 \pm 1.4$ vs. $139.2 \pm 1.2$, $* *: p<0.001)$. the donor cells could migrate to the lesion site (Mothe et al., 2011). In contrast, the transplanted cell sheet remained at the site of transplantation and showed longer survival. Thus, a combination of these two methods has the potential to maximize the therapeutic effects of the HAC-MSCs.

\subsection{Toward clinical application}

Translating preclinical experiments to clinical trial is accompanied by many obstacles, many clinical trials document they are safe but not so effective, and so are MSC clinical trials for stroke, for example. Borlongan (2019) stresses that the importance of strict adherence to successful preclinical experiment protocol especially optimal cell dose, timing of transplantation windows, and transplantation route is key to successful clinical trial. In the case of ALS transplantation, as the aggressive and progressive nature of ALS, future clinical application should be designated to transplant with as many cells of cell sheet as possible, and as early after the diagnosis as possible via the cerebral ventricle and/or (lumbar) spinal cord using endoscopic procedure.

\section{Conclusion}

The cytoprotective effects of HAC-MSC sheets can be utilized not only for chronic diseases such as ALS but also for acute diseases such as spinal cord injury and ischemic stroke. Introduction of other trophic factors or key proteins into the HAC vector could extend the therapeutic applications without limitation. In order to further enhance the therapeutic effects, it will be necessary to enhance the cytoprotective potency of donor cells using cell engineering technology, and the HAC system has great potential in this regard.

\section{Materials and methods}

\subsection{Animals}

Mice expressing high levels of mutant $\mathrm{Cu} / \mathrm{Zn}$ superoxide dismutase (SOD1), B6SJL-Tg (SOD1*G93A) 1Gur/J mice, or SOD1 ${ }^{\text {G93A }}$ mice, were obtained from Jackson Lab (Bar Harbor, ME, USA). Male SOD1 ${ }^{\text {G93A }}$ mice were crossed with wild-type C57BL/6CR mice (Shimizu Laboratory Supplies, Kyoto, Japan) and the lineage was maintained on a C57BL/ 6 background. The experimental procedures were approved by the Tottori University animal experiment committee (h30-Y-024).

\subsection{HAC-MSCs}

The HAC-MSCs also express reporter proteins: green fluorescent protein (GFP), Emerald luciferase (E-Luc; Toyobo, Osaka, Japan), and human neurotrophic factors (Watanabe et al., 2015). The cells were maintained in Dulbecco's Modified Eagle's medium (DMEM, high glucose; D6429, Sigma-Aldrich, Tokyo, Japan), $0.4 \%$ penicillin- 


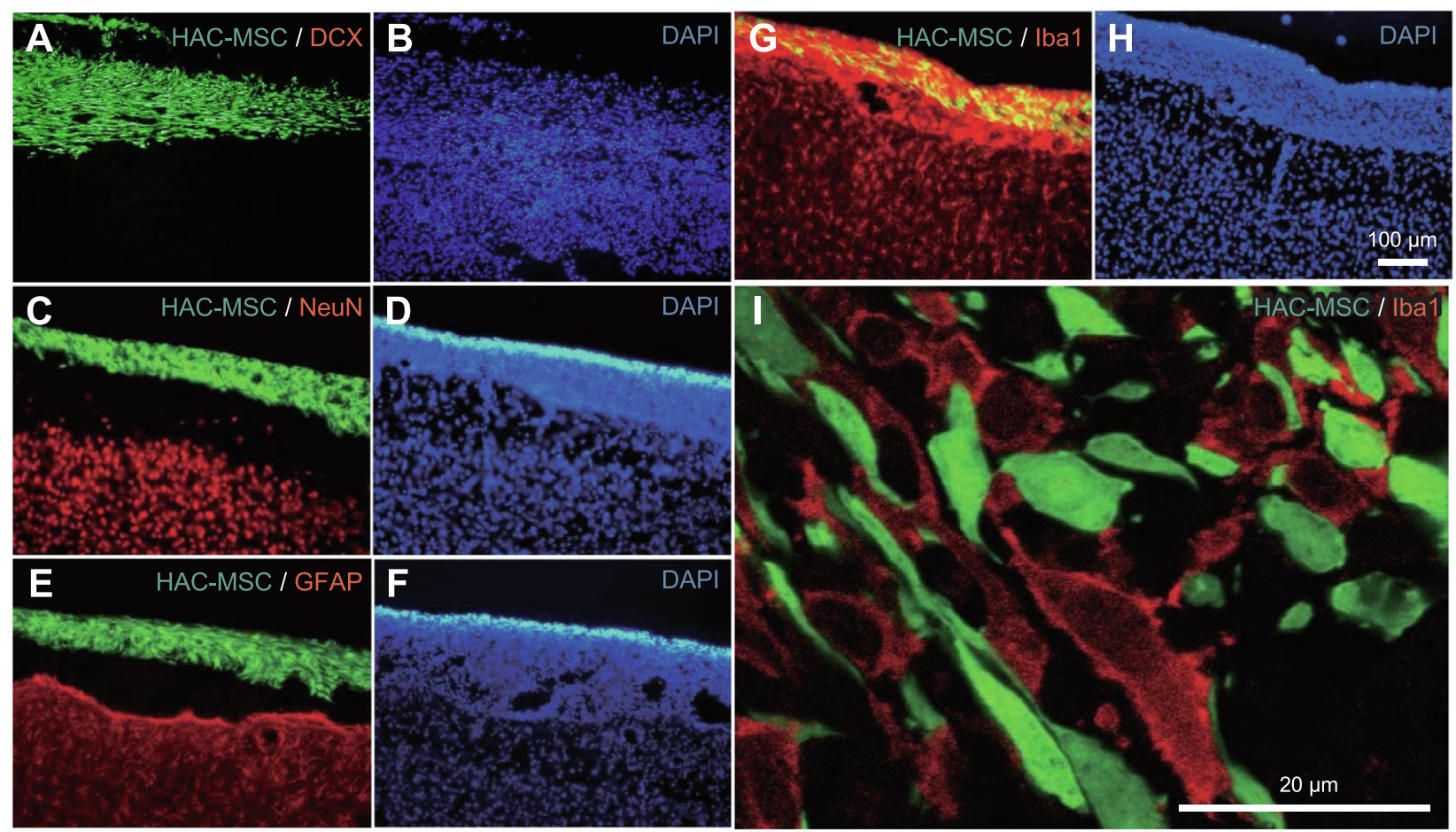

Fig. 5. Expression of neural cell markers in donor cells. Seven days after transplantation the expression of neural cell markers in the transplantation region was examined by immunohistochemistry (DCX: A; NeuN: C; GFAP: E; Iba1: G and I). DAPI staining was also performed to examine the distribution of cell nuclei (B, D, F, H). HAC-MSCs were negative for DCX (A), NeuN (C) and GFAP (E). Immunostaining, at first glance, seemed to show co-localization of Iba1 (E, red) and HAC-MSCs (green). When this region was observed at higher magnification (I), the Iba1 and HAC-MSC signals did not directly overlap suggesting that the host Iba1-positive cells had infiltrated into the layers of the HAC-MSC sheet.
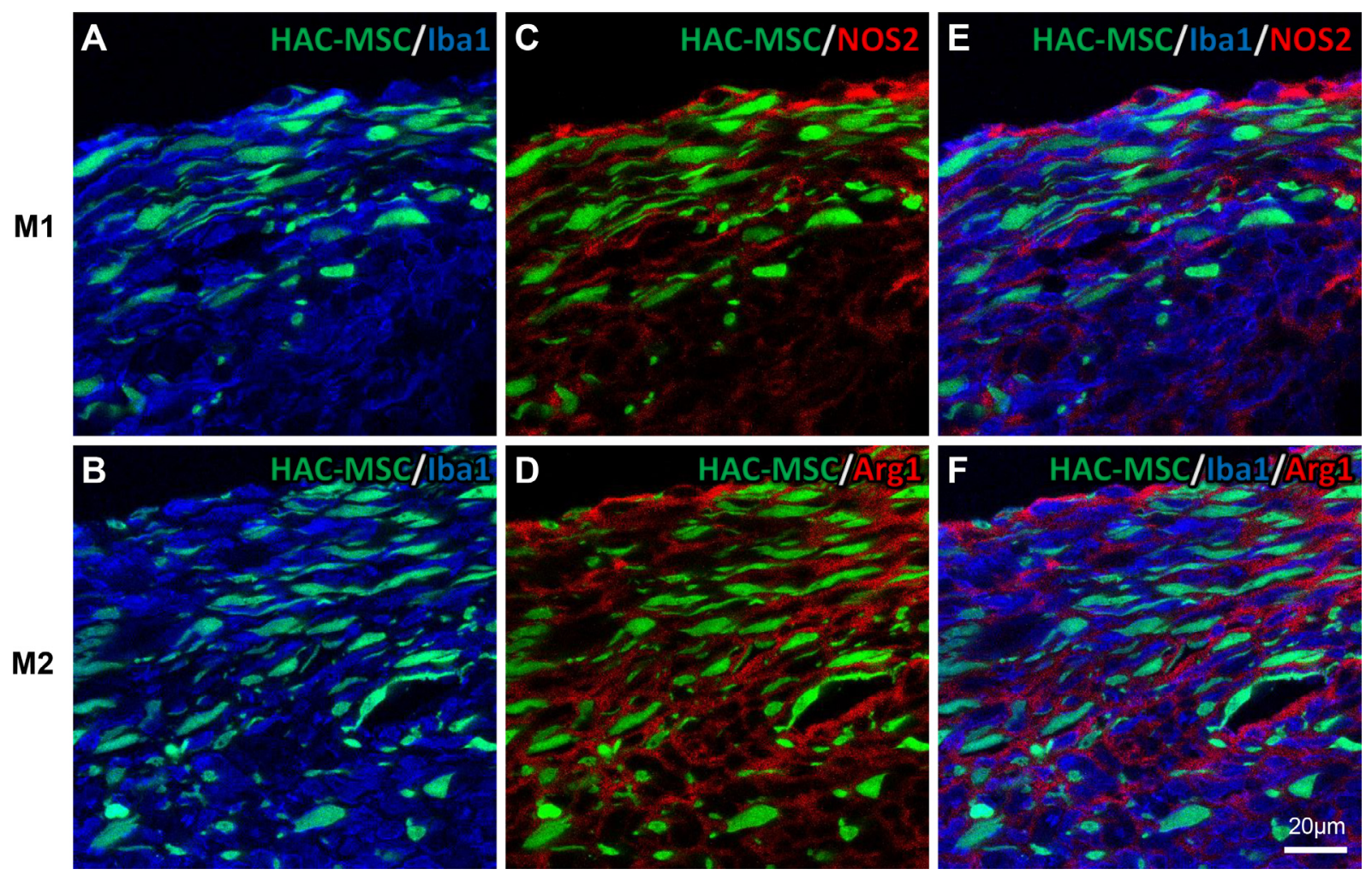

Fig. 6. HAC-MSC transplantation triggers Microglial activation. To examine the phenotype of infiltrated microglia, we performed Immunohistochemistry on the sections of the sheet-transplanted region of B6 mice on day 7 (A, B). Each sample was stained with anti-Iba1 antibody and also with anti-NOS2 antibody (A) or antiArg-1 antibody (B). Anti-Iba1 antibody was detected by Cruz flour 405 labelled secondary antibody (blue). Anti-NOS2 and anti-Arg1 antibodies were detected by CF633 labelled secondary antibody (red). High-magnification confocal microscopy showed these infiltrated microglias are partly Arg-1 positive M2 phenotype cells but some population was NOS2 positive M1 marker positive. 
streptomycin (Wako, Osaka, Japan), 10\% fetal bovine serum (FBS; FB136, biosera, Tokyo, Japan), and $4 \mu \mathrm{g} / \mathrm{ml}$ blasticidin (Funakoshi, Tokyo, Japan), and cells were cultured at $37{ }^{\circ} \mathrm{C}$ with $5 \% \mathrm{CO}_{2}$.

\subsection{Preparation of HAC-MSC sheets}

Cell sheets were prepared using UpCell 48 multi-well dishes (CellSeed Inc., Tokyo, Japan) according to the manufacturer's protocol. Each well $\left(1.1 \mathrm{~cm}\right.$ in diameter) was coated with FBS at $37^{\circ} \mathrm{C}$ overnight, then the HAC-MSCs were seeded onto each well at about $2 \times 10^{5}$ cells/ well, and cultured until they reached confluence. At this point, the dish was placed at room temperature (approximately $25^{\circ} \mathrm{C}$ ) for $30 \mathrm{~min}$, and the HAC-MSCs were retrieved as a cell sheet of approximately $5 \mathrm{~mm}$ in diameter due to shrinkage after exfoliation.

\subsection{Transplantation}

The immunosuppressant FK506 (kindly provided by Astellas Pharma, Tokyo, Japan) was dissolved in the drinking water and administered ( $3 \mathrm{mg} / \mathrm{kg}$ BW/day) to mice 1 week prior to operation and was continued until death. For the transplantation procedure, after exposing the mouse skull, a rectangular area of the left cerebral cortex approximately $5 \mathrm{~mm}$ (width) by $4 \mathrm{~mm}$ (lengthwise) was surgically exposed. The dura matter was removed and a sheet of HAC-MSCs was gently placed onto the surface (Fig. 1). The sheet was maintained in place for $5 \mathrm{~min}$ to facilitate adhesion to the host brain. After covering with surgical dura (Gore-Tex, Japan Goa Inc., Tokyo, Japan), the removed skull section was returned. The bone gap was filled with gauze, and the skull was fixed with dental resin (Shofu, Kyoto, Japan). The incised skin was then sutured, and the wound area was coated with gentacin (MSD, Tokyo, Japan). For the control group, sham surgery was performed by exposing the brain surface without application of a cell sheet.

Prior to intrathecal injection, an immunosuppressant was administered as described above. For the cell injection, an injection needle was stereotaxically inserted at a caudal position $6 \mathrm{~mm}$ from the Bregma suture on the midline to $7.5 \mathrm{~mm}$ depth from the brain surface, as described previously (Morita et al., 2008). Twenty-five $\mu$ lof the cell suspension was injected under pressure using a syringe pump (100 ml/ min, $1 \times 10^{6}$ cells $/ 25 \mu \mathrm{l}$ ).

\subsection{Bioluminescence imaging (BLI)}

For the in vitro analysis, a predetermined number of HAC-MSCs was seeded onto a 96-well plate. D-luciferin (Promega, Madison, WI, USA) was added to each well $(0.1 \mathrm{mg} / \mathrm{ml}$ in phosphate buffered saline [PBS]). Emission values were measured 3 min after the addition of D-luciferin (exposure time of $10 \mathrm{~s}$ ).

For in vivo observation, cells were transplanted into mice as a sheet or through the fourth ventricle, and the emission signals were monitored at 4 time points: immediately after transplantation, and 3, 7, and 14 days post-operation. The mice were anesthetized with $2 \%$ isoflurane (Fallen; AbbVie LLC, Tokyo, Japan) and 100\% $\mathrm{O}_{2}$. D-luciferin (150 mg/ $\mathrm{kg}$ BW) in PBS was intraperitoneally administered. For the cell sheet transplantation, the signals were measured $15 \mathrm{~min}$ after administration (exposure time of $1 \mathrm{~min}$ ). For the intrathecal injection, the measurement was performed $30 \mathrm{~min}$ after administration (exposure time of 5 min).

For both the in vitro and vivo experiments, the bioluminescence emitted from the HAC-MSCs was measured using IVIS Spectrum ver. 4.0 (Summit Pharmaceuticals International Corporation [SPI], Tokyo, Japan), and analyzed using IVIS Live image 2.60 (SPI) software. The region of interest (ROI) was set within the transplanted area. An adjacent non-transplanted area was also measured to obtain a background value. The value obtained by subtracting the background value from the ROI value was defined as the luminescence value.

\subsection{Immunohistochemical staining}

The HAC-MSC sheet was transplanted into wild-type mice at 100 days of age, and tissues were obtained 7 days after transplantation. Animals were deeply anesthetized with pentobarbital (Somnopentil; Kyoritsu Pharma, Nara, Japan) and transcardially perfused with saline, followed by $4 \%$ paraformaldehyde. Brains were removed, fixed in $4 \%$ paraformaldehyde for $18 \mathrm{~h}$, and then immersed in $30 \%$ sucrose in PBS at $4{ }^{\circ} \mathrm{C}$ for a minimum of 3 days. Using a microtome (Leica, Eisfeld, Germany), frozen sections of $30 \mu \mathrm{m}$ thickness were prepared. Sections were blocked with $5 \%$ bovine serum albumin (Sigma-Aldrich) in PBS for $2 \mathrm{~h}$ at room temperature and the primary antibody reaction was performed at $4{ }^{\circ} \mathrm{C}$ overnight. Sections were incubated in secondary antibody at room temperature for $3 \mathrm{~h}$, then mounted with Vectashield containing 4', 6-diamidino-2-phenylindole (DAPI; Vector Laboratories, Burlingame, CA, USA). Stained sections were observed using a BX53 fluorescence microscope (Olympus, Tokyo, Japan), or a TCS SP2 confocal microscope (Leica). The following primary antibodies were used: goat polyclonal antibody against doublecortin (DCX) (1:250; Santa Cruz Biotechnology, CA, USA), rabbit anti-NeuN monoclonal antibody (1:500; abcam, Cambridge, UK), rabbit polyclonal antibody against GFAP (1:500; abcam), rabbit anti-Iba1 antibody (1:1000; Wako), mouse anti-NOS2 antibody (1:250; Santa Cruz), and mouse anti-Arginase1 (Arg1) antibody (1:250; Santa Cruz). As second antibodies, donkey antirabbit IgG Texas red (TR; 1:100; Santa Cruz), donkey anti-mouse IgG CF633 (1:100; Santa Cruz), anti-rabbit IgG CFL405, and donkey antigoat IgG Alexa flor 594 (1:100; Abcam) were used.

\subsection{Western blotting}

Mice were sacrificed by intraperitoneal injection of pentobarbital. The brains were rapidly removed and immediately stored in liquid nitrogen until use. The cerebral cortex was homogenized with 10 volumes of RIPA buffer ( $\mathrm{pH} 8.0 ; \mathrm{NaCl} 150 \mathrm{mM}$, Triton X-100 1\%, Sodium deoxycholate $0.5 \%$, SDS $0.1 \%$, and Tris- $\mathrm{HCl} 50 \mathrm{mM}$ ) with a protease inhibitor cocktail (Complete Mini; Roche Diagnostics, Tokyo, Japan). The samples were separated on $12.5 \%$ SDS-polyacrylamide gels (Super Sep Ace; Wako) and transferred onto a transfer membrane (Immobilon; Millipore, MA, USA). Chemiluminescence detection was performed using ECL Prime (GE Healthcare, IL, USA). The following primary antibodies were used: mouse anti- $\alpha$-tubulin monoclonal antibody (1:1000; Sigma), rabbit anti-Akt antibody (1:1000; Cell Signaling, MA, USA) which represents total Akt, and rabbit anti-phosphorylated-Akt (Ser473) antibody (1:1000; Cell Signaling). The following secondary antibodies were used: ECL anti-rabbit IgG from donkey, and anti-mouse IgG from donkey (1:10,000; GE Healthcare).

\subsection{Evaluation of mice}

Age of disease onset, day of death, disease duration, body weight, and hindlimb reflex score were evaluated. After transplantation, the body weight and hindlimb reflex score was measured once every week. The hindlimb reflex score was a 5-point evaluation $(2,1.5,1,0.5$, and 0 ) with 2 being normal and 0 being completely rigid, and the average value of each limb was used for evaluation. Disease onset was determined as described in our previous report (Watanabe et al., 2015).

\subsection{Statistical analysis}

Data are expressed as the mean \pm standard error (SE) and calculated using a Student's T test or the Kaplan-Meier method (log-rank test). Statistical significance was set at a $p$ value of $<0.05$.

\section{Declaration of Competing Interest}

The authors declare that they have no known competing financial 
interests or personal relationships that could have appeared to influence the work reported in this paper.

\section{Acknowledgements}

The authors thank junior associate Prof. takao Mukuta (department of anatomy faculty of medicine, Tottori University) for valuable suggestion in the immunohistochemical experiment. This research was partly performed at the Tottori Bio Frontier managed by Tottori prefecture.

\section{Funding}

This work was supported by JSPS KAKENHI Grant Numbers JP16K20011 (MN) and JP16K09719 (YW).

\section{Appendix A. Supplementary data}

Supplementary data to this article can be found online at https:// doi.org/10.1016/j.brainres.2019.146444.

\section{References}

Acosta, S.A., Tajiri, N., Hoover, J., Kaneko, Y., Borlongan, C.V., 2015. Intravenous bone marrow stem cell grafts preferentially migrate to spleen and abrogate chronic inflammation in stroke. Stroke 46, 2616-2627.

Blondheim, N.R., Levy, Y.S., Ben-Zur, T., Burshtein, A., Cherlow, T., Kan, I., Barzilai, R., Bahat-Stromza, M., Barhum, Y., Bulvik, S., Melamed, E., Offen, D., 2006. Human mesenchymal stem cells express neural genes, suggesting a neural predisposition. Stem Cells Dev. 15, 141-164.

Borlongan, C.V., 2019. Concise review: stem cell therapy for stroke patients: are we there yet? Stem. Cells Transl. Med.

Chen, D., Fu, W., Zhuang, W., Lv, C., Li, F., Wang, X., 2017. Therapeutic effects of intranigral transplantation of mesenchymal stem cells in rat models of Parkinson's disease. J. Neurosci. Res. 95, 907-917.

Chen, G., Qi, Y., Niu, L., Di, T., Zhong, J., Fang, T., Yan, W., 2015. Application of the cell sheet technique in tissue engineering. Biomed. Rep. 3, 749-757.

Coatti, G.C., Beccari, M.S., Olavio, T.R., Mitne-Neto, M., Okamoto, O.K., Zatz, M., 2015. Stem cells for amyotrophic lateral sclerosis modeling and therapy: myth or fact? Cytometry A 87, 197-211.

De Vocht, N., Praet, J., Reekmans, K., Le Blon, D., Hoornaert, C., Daans, J., Berneman, Z., Van der Linden, A., Ponsaerts, P., 2013. Tackling the physiological barriers for successful mesenchymal stem cell transplantation into the central nervous system. Stem Cell Res. Ther. 4, 101.

Endo, F., Komine, O., Fujimori-Tonou, N., Katsuno, M., Jin, S., Watanabe, S., Sobue, G., Dezawa, M., Wyss-Coray, T., Yamanaka, K., 2015. Astrocyte-derived TGF-beta1 accelerates disease progression in ALS mice by interfering with the neuroprotective functions of microglia and T cells. Cell Rep. 11, 592-604.

Foudah, D., Redondo, J., Caldara, C., Carini, F., Tredici, G., Miloso, M., 2012. Expression of neural markers by undifferentiated rat mesenchymal stem cells. J. Biomed. Biotechnol. 2012, 820821.

Foudah, D., Redondo, J., Caldara, C., Carini, F., Tredici, G., Miloso, M., 2013. Human mesenchymal stem cells express neuronal markers after osteogenic and adipogenic differentiation. Cell. Mol. Biol. Lett. 18, 163-186.

Gnecchi, M., Danieli, P., Malpasso, G., Ciuffreda, M.C., 2016. Paracrine mechanisms of mesenchymal stem cells in tissue repair. Methods Mol. Biol. 1416, 123-146.

Gothelf, Y., Abramov, N., Harel, A., Offen, D., 2014. Safety of repeated transplantations of neurotrophic factors-secreting human mesenchymal stromal stem cells. Clin. Transl. Med. 3, 21.

Henriques, A., Pitzer, C., Schneider, A., 2010. Neurotrophic growth factors for the treatment of amyotrophic lateral sclerosis: where do we stand? Front. Neurosci. 4, 32.
Hsuan, Y.C., Lin, C.H., Chang, C.P., Lin, M.T., 2016. Mesenchymal stem cell-based treatments for stroke, neural trauma, and heat stroke. Brain Behav. 6, e00526.

Ito, M., Shichinohe, H., Houkin, K., Kuroda, S., 2017. Application of cell sheet technology to bone marrow stromal cell transplantation for rat brain infarct. J. Tissue Eng. Regener. Med. 11, 375-381.

Joyce, N., Annett, G., Wirthlin, L., Olson, S., Bauer, G., Nolta, J.A., 2010. Mesenchymal stem cells for the treatment of neurodegenerative disease. Regener. Med. 5, 933-946.

Lescaudron, L., Unni, D., Dunbar, G.L., 2003. Autologous adult bone marrow stem cell transplantation in an animal model of huntington's disease: behavioral and morphological outcomes. Int. J. Neurosci. 113, 945-956.

Lunn, J.S., Hefferan, M.P., Marsala, M., Feldman, E.L., 2009. Stem cells: comprehensive treatments for amyotrophic lateral sclerosis in conjunction with growth factor delivery. Growth Fact. 27, 133-140.

Mazzini, L., Ferrero, I., Luparello, V., Rustichelli, D., Gunetti, M., Mareschi, K., Testa, L., Stecco, A., Tarletti, R., Miglioretti, M., Fava, E., Nasuelli, N., Cisari, C., Massara, M., Vercelli, R., Oggioni, G.D., Carriero, A., Cantello, R., Monaco, F., Fagioli, F., 2010. Mesenchymal stem cell transplantation in amyotrophic lateral sclerosis: a Phase I clinical trial. Exp. Neurol. 223, 229-237.

Miyahara, Y., Nagaya, N., Kataoka, M., Yanagawa, B., Tanaka, K., Hao, H., Ishino, K., Ishida, H., Shimizu, T., Kangawa, K., Sano, S., Okano, T., Kitamura, S., Mori, H., 2006. Monolayered mesenchymal stem cells repair scarred myocardium after myocardial infarction. Nat. Med. 12, 459-465.

Morita, E., Watanabe, Y., Ishimoto, M., Nakano, T., Kitayama, M., Yasui, K., Fukada, Y., Doi, K., Karunaratne, A., Murrell, W.G., Sutharsan, R., Mackay-Sim, A., Hata, Y., Nakashima, K., 2008. A novel cell transplantation protocol and its application to an ALS mouse model. Exp. Neurol. 213, 431-438.

Mothe, A.J., Bozkurt, G., Catapano, J., Zabojova, J., Wang, X., Keating, A., Tator, C.H., 2011. Intrathecal transplantation of stem cells by lumbar puncture for thoracic spinal cord injury in the rat. Spinal Cord. 49, 967-973.

Oh, K.W., Moon, C., Kim, H.Y., Oh, S.I., Park, J., Lee, J.H., Chang, I.Y., Kim, K.S., Kim, S.H., 2015. Phase I trial of repeated intrathecal autologous bone marrow-derived mesenchymal stromal cells in amyotrophic lateral sclerosis. Stem Cells Transl. Med. 4, 590-597.

Ouyang, H.W., Cao, T., Zou, X.H., Heng, B.C., Wang, L.L., Song, X.H., Huang, H.F., 2006 Mesenchymal stem cell sheets revitalize nonviable dense grafts: implications for repair of large-bone and tendon defects. Transplantation 82, 170-174.

Sampaio, T.B., Savall, A.S., Gutierrez, M.E.Z., Pinton, S., 2017. Neurotrophic factors in Alzheimer's and Parkinson's diseases: implications for pathogenesis and therapy. Neural Regener. Res. 12, 549-557.

Sart, S., Ma, T., Li, Y., 2014. Preconditioning stem cells for in vivo delivery. BioResearch open access 3, 137-149.

Sykova, E., Rychmach, P., Drahoradova, I., Konradova, S., Ruzickova, K., Vorisek, I., Forostyak, S., Homola, A., Bojar, M., 2017. Transplantation of mesenchymal stromal cells in patients with amyotrophic lateral sclerosis: results of phase I/IIa clinical trial. Cell Transpl. 26, 647-658.

Thomsen, G.M., Gowing, G., Latter, J., Chen, M., Vit, J.P., Staggenborg, K., Avalos, P., Alkaslasi, M., Ferraiuolo, L., Likhite, S., Kaspar, B.K., Svendsen, C.N., 2014. Delayed disease onset and extended survival in the SOD1G93A rat model of amyotrophic lateral sclerosis after suppression of mutant SOD1 in the motor cortex. J. Neurosci. 34, 15587-15600.

Toyoshima, A., Yasuhara, T., Kameda, M., Morimoto, J., Takeuchi, H., Wang, F., Sasaki, T., Sasada, S., Shinko, A., Wakamori, T., Okazaki, M., Kondo, A., Agari, T., Borlongan, C.V., Date, I., 2015. Intra-Arterial transplantation of allogeneic mesenchymal stem cells mounts neuroprotective effects in a transient ischemic stroke model in rats: analyses of therapeutic time window and its mechanisms. PLoS One 10, e0127302.

Vercelli, A., Mereuta, O.M., Garbossa, D., Muraca, G., Mareschi, K., Rustichelli, D., Ferrero, I., Mazzini, L., Madon, E., Fagioli, F., 2008. Human mesenchymal stem cell transplantation extends survival, improves motor performance and decreases neuroinflammation in mouse model of amyotrophic lateral sclerosis. Neurobiol. Dis. 31, 395-405.

Watanabe, Y., Kazuki, Y., Kazuki, K., Ebiki, M., Nakanishi, M., Nakamura, K., Yoshida Yamakawa, M., Hosokawa, H., Ohbayashi, T., Oshimura, M., Nakashima, K., 2015. Use of a human artificial chromosome for delivering trophic factors in a rodent model of amyotrophic lateral sclerosis. Mol. Ther. Nucleic Acids 4, e253.

Zhang, Y., Yu, S., Tuazon, J.P., Lee, J.Y., Corey, S., Kvederis, L., Kingsbury, C., Kaneko, Y., Borlongan, C.V., 2019. Neuroprotective effects of human bone marrow mesenchymal stem cells against cerebral ischemia are mediated in part by an anti-apoptotic mechanism. Neural Regener. Res. 14, 597-604. 